\title{
Predictive Factors of Tumor Recurrence and Survival in Patients with Hepatocellular Carcinoma treated with Transarterial Chemoembolization
}

\author{
Razvan Cerban ${ }^{1}$, Carmen Ester ${ }^{1}$, Speranta Iacob ${ }^{1}$, Mugur Grasu², Liliana Pâslaru ${ }^{3}$, Radu Dumitru ${ }^{2}$, Ioana Lupescu², \\ Georgiana Constantin ${ }^{3}$, Adina Croitoru ${ }^{4}$, Liana Gheorghe ${ }^{1}$
}

\author{
1) Center for Digestive \\ Diseases and Liver \\ Transplantation, Fundeni \\ Clinical Institute; Carol Davila \\ University of Medicine and \\ Pharmacy, Bucharest, \\ 2) Center for Interventional \\ Radiology and Medical \\ Imaging, Fundeni Clinical \\ Institute; Carol Davila \\ University of Medicine and \\ Pharmacy, Bucharest, \\ 3) Department of \\ Biochemistry, Fundeni \\ Clinical Institute, Bucharest, \\ 4) Department of Medical \\ Oncology, Fundeni Clinical \\ Institute, \\ Bucharest, Romania
}

\begin{abstract}
Background \& Aims: To evaluate the predictive factors for recurrence of the disease and overall survival (OS) after achieving complete response (CR) in patients with hepatocellular carcinoma (HCC) treated with transarterial chemoembolization (TACE).

Methods: From January 2013 to December 2017, 168 treatment-naïve patients diagnosed with HCC underwent TACE as a first-line therapy and the gathered data was retrospectively reviewed. We determined the predictive factors for complete response (CR), for recurrence after CR and for survival using the Cox proportional hazard model.

Results: Median follow-up was 27.4 months (range 4-65 months). The mean patient age was $62.2 \pm 7.9$ years. Eighty-three patients had an $\alpha$-fetoprotein (AFP) level $>20 \mathrm{ng} / \mathrm{mL}$. The median maximal diameter of the tumors was $3.5 \mathrm{~cm}$. Sixty-three patients (37.5\%) achieved CR after TACE, and recurrence after CR was detected in 37 patients $(58.7 \%)$. In multivariate analysis, tumor size $(\leq 4.5 \mathrm{~cm})$ and a single tumor were found to be predictive factors for $\mathrm{CR}$, with hazard ratios (HRs) of 2.352 ( $\mathrm{p}=0.022$ ) and $3.964(\mathrm{p}<0.0001)$, respectively. After achieving CR the median time to recurrence was 12 months (range 6-24 months). Elevated serum AFP $>25$ $\mathrm{ng} / \mathrm{mL}$ and multiple tumors were demonstrated to have a significant relationship with recurrence after CR, with HRs of $1.650(\mathrm{p}=0.05)$ and $3.932(\mathrm{p}=0.038)$, respectively. Increased initial serum AFP $>22 \mathrm{ng} / \mathrm{mL}$, tumor size $>4.5 \mathrm{~cm}$, outside Milan criteria, not receiving a liver transplant and presence of portal vein thrombosis (PVT) were correlated with poor survival.

Conclusions: In patients treated with TACE as an initial therapy, tumor size $(\leq 4.5 \mathrm{~cm})$ and single tumor were predictive factors for CR. Multiple nodules and an elevated serum AFP $>25 \mathrm{ng} / \mathrm{mL}$ were predictive factors for recurrence after CR. Outside Milan criteria tumors, elevated AFP levels and the presence of PVT were significantly correlated with decreased survival.
\end{abstract}

Key words: hepatocellular carcinoma - transarterial chemoembolization - alpha-fetoprotein - tumor recurrence - survival.

Abbreviations: AFP: alpha-fetoprotein; ALT: alanine aminotransferase; AST: aspartate aminotransferase; ART: assessment for retreatment with transarterial chemoembolization; BCLC: Barcelona Clinic Liver Cancer; CR: complete response; DEBDOX: Doxorubicin-loaded DC Bead; GGT: gammaglutamyltransferase; HCC: hepatocellular carcinoma; HBV: hepatitis B virus; HCV: hepatitis C virus; HR: hazard ratio; MELD: model for end-stage liver disease; mRECIST: modified Response Evaluation Criteria in Solid Tumors; PVT: portal vein thrombosis; RFA: radiofrequency ablation; OS: overall survival; TACE: transarterial chemoembolization.

\section{INTRODUCTION}

Received: 12.09 .2019

Accepted: 30.11 .2018
The most widely utilized staging system for HCC is the

Hepatocellular carcinoma (HCC) is the third leading cause of cancer-related death worldwide. It is responsible for more than 500,000 deaths with over 600,000 new cases yearly worldwide [1].
Barcelona Clinic Liver Cancer (BCLC) staging system [2]. This system distinguishes five tumor stages, which are associated with different treatment options.

The treatment modalities that are used today are various, depending on the stage of HCC and the severity of the underlying liver disease. Complete cure of both HCC and liver disease can be achieved only through liver transplantation, but 
unfortunately, due to the specific selection criteria and the low availability of liver grafts, this treatment can be performed in a limited number of patients [3].

It is estimated that $70 \%$ of patients with HCC are diagnosed at intermediate (BCLC stage B) or advanced (BCLC stage C) tumor stage, with limited treatment options [4].

For patients with large $(>5 \mathrm{~cm})$ or multifocal HCC without macrovascular invasion or extrahepatic metastasis, Child Pugh scores $<8$ and a performance status of 0 , the most widely used treatment is transarterial chemoembolization (TACE). It is considered the first line treatment recommended for intermediate HCC (BCLC stage B), based on several randomized clinical trials and meta-analysis $[5,6]$. Transarterial chemoembolization is a major type of transarterial therapies that consists of injecting anticancer drugs into the feeding arteries of a tumor along with particles designed to slow or stop the further arterial supply of oxygen and nutrients to achieve ischemia and necrosis of that tumor. It leads to survival benefits in the majority of patients, without affecting the liver functional reserve. Hepatocellular carcinoma patients treated with TACE showed survival rates of $70.3 \%$ at 1 year, $51.8 \%$ at 2 years, $40.4 \%$ at 3 years, and $32.4 \%$ at 5 years [7].

This procedure can also be used when curative treatments such as radiofrequency ablation (RFA) or surgical resection are either unsuccessful or unfeasible in patients with early HCC. Based on clinical decision, in real life practice, approximately $40 \%$ of TACE procedures are performed in patients with early stage HCC [8]. Overall survival (OS) rates in patients with early-stage HCC in whom TACE was performed were $91 \%$ at 1 year and $52 \%$ at 5 years; serum albumin, tumor size, tumor number and recurrence interval were demonstrated to influence mortality [9].

The aim of this study was to establish the independent predictive factors associated with disease recurrence after complete response (CR) and overall survival (OS) after TACE in HCC patients at a single university hospital.

\section{METHODS}

\section{Patients}

One hundred sixty eight patients with HCC who received TACE as initial treatment were included in the study. The TACE was performed at the Fundeni Clinical Institute, between January 2013 and December 2017.

All patients were newly diagnosed with HCC, without any previous HCC treatment and without extrahepatic spread at the time of their diagnosis. Hepatocellular carcinoma was diagnosed based on histological findings or typical imaging features as defined by the EASL and AASLD guidelines [10]. Tumor staging was determined according to the BCLC staging system [11]. Portal vein thrombosis (PVT) was evaluated according to previously published criteria [12] and only patients with nonmalignant thrombosis were included. The included patients had no indication for surgical resection or refused surgery as initial treatment. Other inclusion criteria were: patients with HCC older than 18 years and expected survival time longer than 6 months; without history of previous HCC therapy or liver transplantation and Eastern Cooperative Oncology Group (ECOG) performance status of 0-1.
The exclusion criteria were: extensive tumors in the hepatic lobes, decompensated liver cirrhosis, poor overall health status, unable to tolerate the procedure, impaired renal function (creatinine $>2 \mathrm{mg} / \mathrm{dl}$ ), severe heart and lung disease, extrahepatic metastasis and malignant portal vein thrombosis.

Detailed clinical and laboratory information at the time of diagnosis was collected for all the patients, including liver biochemistries: aspartate aminotransferase (AST), alanine aminotransferase (ALT), gamma-glutamyltransferase (GGT), alkaline phosphatase, total and direct bilirubin, albumin, platelet count, INR, creatinine, hepatitis $B$ virus (HBV), hepatitis $\mathrm{C}$ virus $(\mathrm{HCV})$ and serum alphafetoprotein (AFP).

\section{Therapeutic and response-evaluation protocol}

All patients underwent complete blood count, biochemistry, blood type, blood coagulation, liver function, renal function, ultrasound examination and computer tomography (CT) or magnetic resonance imaging (MRI) examination and upper endoscopy for esophageal varices evaluation. In 9 patients (5.3\%) who were found to have high-risk esophageal varices an endoscopic band ligation was performed and the TACE procedure was postponed for 4 weeks.

All TACE procedures were performed in our center by two experienced interventional radiologists. The conventional TACE (cTACE) was performed using a solution of $50 \mathrm{mg}$ doxorubicin hydrochloride in a 5-20 ml mixed solution of lipiodol and contrast agent. Subsequently, embolization was performed using gelatin sponge particles until the entire tumor blood supply artery was blocked and tumor staining vanished completely. In patients who underwent Doxorubicin-loaded DC Bead (DEBDOX), TACE was done using DC Beads ${ }^{\circledast}$ particles (Biocompatibles, Surrey, United Kingdom) loaded with doxorubicin hydrochloride.

The radiological response was evaluated at 4 to 6 weeks after TACE using multislice CT scanner or MRI. The tumor marker response was also determined. The images were analyzed by two senior radiologists and any discordant opinion required a third evaluation.

All patients included in the study were evaluated according to the modified Response Evaluation Criteria in Solid Tumors (mRECIST) [13]. Dense compact lipiodolization with complete disappearance of any intratumoral enhancement in the tumor at CT scan, or no enhancement of the nodule on MRI was considered to be a complete response (CR) [13]. In patients who achieved CR, a follow-up CT scan or MRI at 3 months was performed to detect reccurrence. A plain chest X-ray scan or chest CT were also performed during the follow-up period. For overall survival assessment, patients were followed-up until the closure time of the study, time of death, liver transplant (if performed), or until the last clinical evaluation for patients lost to follow-up.

Repeat session of TACE was performed if CT or MRI identified remaining viable tumor. If no viable HCC was depicted on imaging studies, radiologic evaluation and tumor marker measurement were done every 3 months. Transarterial chemoembolization was discontinued in cases with vascular invasion, poor liver function, severe adverse effects or tumor progression. 


\section{Predictive factors}

In order to identify predictive factors associated with CR, recurrence after TACE-induced CR and overall survival (OS), the following baseline parameters were analyzed: age, gender, hepatitis $\mathrm{B}(\mathrm{HBV})$ and $\mathrm{C}(\mathrm{HCV})$ viruses, model for end-stage liver disease (MELD) score, serum AFP value, size and number of tumors, type of TACE treatment (cTACE or DEBDOX) and portal vein thrombosis (PVT).

\section{Ethical statement}

The study design was reviewed and approved by the Fundeni Clinical Institute, Ethical Committee. All patients signed an informed consent in written form before they underwent diagnostic and therapeutic procedure at the time of hospitalization according to our institutional guidelines. This study complied with the guidelines and principles of the declaration of Helsinki.

\section{Statistical analysis}

Continuous variables were reported as means + SD (standard deviation) or as median (range) when appropriate. Categorical variable were reported as percentages. Univariate analysis was performed to identify the predicting factors. The cut-off values for the variables used in the analysis were selected based on the Youden index. We used the Cox proportional hazards model and a stepwise selection was done using statistically significant variables at univariate analysis ( $\mathrm{p}$ $<0.05$ ), in order to identify independent predictive factors by multivariate analysis. The overall survival rates were calculated using the Kaplan-Meier method and were compared using log rank tests. A two-tailed p-value below 0.05 was regarded as significant difference.

Statistical analyses were performed using Medcalc ver. 13.3.3.0 software.

\section{RESULTS}

\section{Patients}

One hundred sixty eight patients treated with TACE, who met the inclusion criteria were selected for the study. The baseline characteristics of these patients are summarized in Table I. The mean age was $62.2 \pm 7.9$ years. There were 115 males $(68.4 \%)$ and 53 females $(31.5 \%)$. The most common cause of cirrhosis was hepatitis $\mathrm{C}$ virus infection. The median MELD score was 9 (range, 6 to 17), and the median Child Pugh score was 6. The median maximal diameter of the tumors was $3.5 \mathrm{~cm}$ (range, 1.6 to $10.8 \mathrm{~cm}$ ). Thirty-nine patients $(23.2 \%)$ had a single tumor with diameter of up to $30 \mathrm{~mm}$. In those patients, RFA treatment was unfeasible due to tumor location: next to a major vessel in 8 patients $(4.76 \%)$, liver dome in 6 patients $(3.6 \%)$, proximity to the liver hilum in 5 patients $(2.9 \%)$ and thrombocytopenia $\left(<70 \times 10^{3} / \mathrm{L}\right)$ in 20 patients $(11.9 \%)$.

Sixty patients (35.7\%) had a multinodular pattern. Seventyeight patients had an AFP level $>22 \mathrm{ng} / \mathrm{mL}$. Seventeen patients (10.1\%) had non-malignant PVT. The total number of HCC nodules was 279 .

The median follow-up for the studied group was 27.4 months (range 4-65 months).
Table I. Patients and tumors characteristics

\begin{tabular}{|c|c|}
\hline Characteristics & $\mathrm{n}=168$ patients \\
\hline Age, $($ mean $\pm S D)$ years & $62.2 \pm 7.9$ \\
\hline Gender, n (male) & $115(68.4 \%)$ \\
\hline \multicolumn{2}{|l|}{ Etiology } \\
\hline $\mathrm{HBV}$ & $50(29.7 \%)$ \\
\hline $\mathrm{HCV}$ & $98(58.3 \%)$ \\
\hline Alcohol & $18(10.7 \%)$ \\
\hline Others & $2(1.2 \%)$ \\
\hline Antiviral treatment before HCC & $14(8.3 \%)$ \\
\hline \multicolumn{2}{|l|}{ Child-Pugh class } \\
\hline A & $165(98.2 \%)$ \\
\hline B & $3(1.78 \%)$ \\
\hline Platelet, $\mathrm{x} 10^{3} / \mathrm{L}$ & $104.4 \pm 49.9$ \\
\hline Creatinine, $\mathrm{mg} / \mathrm{dL}$ & $1 \pm 0.8$ \\
\hline Albumin, g/L & $3.6 \pm 0.5$ \\
\hline Bilirubin, mg/dL & $1.3 \pm 0.6$ \\
\hline ALT, UI/L & $54 \pm 59.3$ \\
\hline INR & $1.2 \pm 0.2$ \\
\hline \multicolumn{2}{|l|}{ Tumor number } \\
\hline 1 & $108(64.2 \%)$ \\
\hline 2 & $31(18.4 \%)$ \\
\hline 3 & $12(7.1 \%)$ \\
\hline$>3$ & $17(10.1 \%)$ \\
\hline Tumor size, $($ mean $\pm \mathrm{SD}) \mathrm{cm}$ & $3.9 \pm 1.9$ \\
\hline Tumor size $(>4.5 \mathrm{~cm})$ & $48(28.5 \%)$ \\
\hline BCLC 0 & $4(2.4 \%)$ \\
\hline BCLC A & $110(65.5 \%)$ \\
\hline BCLC B & $54(32.1 \%)$ \\
\hline Nodularity (multinodular) & $60(35.7 \%)$ \\
\hline AFP, ng/ml & $199.8(3.2-5280)$ \\
\hline
\end{tabular}

ALT: alanine transaminases; AFP: alpha-fetoprotien; BCLC: Barcelona Clinic Liver Cancer; HBV: hepatitis B virus; HCV: hepatitis C virus.

\section{Tumor response}

Among 168 HCC patients, 63 patients (37.5\%) achieved CR (Table II) being followed-up for 26 months (range 4-62 months). Hepatocellular carcinoma recurrence after CR was detected in 37 patients (Fig. 1).

In univariate analysis, serum AFP $<22 \mathrm{ng} / \mathrm{mL}$, tumor size $(<4.5 \mathrm{~cm})$, single nodularity were correlated with CR after TACE. Only tumor size $<4.5 \mathrm{~cm}$ and single nodularity were significantly correlated with CR after TACE in multivariate analysis (Table III). Of 63 patients with CR, 55 patients (87.3\%) had tumor size $\leq 4.5 \mathrm{~cm}$, and only 8 patients $(12.69 \%)$ had a tumor size $>4.5 \mathrm{~cm}$. Fifty-one patients $(80.9 \%)$ had a single nodule and 12 patients (19\%) showed multiple nodules. In our group $69.2 \%$ of patients with a single nodule less than $2 \mathrm{~cm}$ achieved CR and $50.6 \%$ of those with nodules between $2.1 \mathrm{~cm}$ and $5 \mathrm{~cm}(\mathrm{p}=0.66)$.

\section{Recurrence after TACE}

Recurrent HCC was diagnosed in 37 patients (58.3\%) out of the 63 patients with CR. After achieving CR the median 
Table II. TACE procedures and patients outcome

\begin{tabular}{ll}
\hline Characteristics & $\mathrm{n}=168$ patients \\
\hline Treatment & $124(73.8 \%)$ \\
cTACE & $44(26.1 \%)$ \\
DEBDOX & $2.1(1-6)$ \\
TACE procedures per patient, & \\
mean & $33(19.6 \%)$ \\
Liver transplant after TACE & \\
procedure & \\
Treatment response & $63(37.5 \%)$ \\
Complete response & $105(62.5 \%)$ \\
Partial response & $51(30.3 \%)$ \\
Sorafenib treatment after TACE & $27.4(6-65)$ \\
Follow up duration, months & \\
Survival outcome, $\mathrm{n}$ & $66(39.2 \%)$ \\
Dead & $93(55.3 \%)$ \\
Alive & $9(5.4 \%)$ \\
Unknown &
\end{tabular}

DEBDOX: Doxorubicin-loaded DC Bead cTACE: conventional transarterial chemoembolization; TACE: transarterial chemoembolization

follow-up period to recurrence was 12 months (range between 5 to 24 months). There were 32 local recurrences (86.4\%) and 5 distant metastases (13.5\%).

Elevated serum AFP (> $25 \mathrm{ng} / \mathrm{mL}$ ) and multiple nodules were significantly associated with tumor recurrence after initial CR in univariate as well as multivariate analysis (Table IV). Patients with multiple tumors (11/12 patients) had a higher recurrence rate than those with single nodule (26/51 patients) (91.6\% vs. 50.9\%; p=0.02). Patients with elevated serum AFP level (> $25 \mathrm{ng} / \mathrm{mL})$ (18/23 patients) had a higher recurrence rate than those with a normal serum AFP level $(\leq 25 \mathrm{ng} / \mathrm{mL})$ (19/40 patients) (78.2\% vs. $47.5 \% ; \mathrm{p}=0.03)$.

\section{Patient survival after TACE}

The median follow-up of the entire study population was 27.4 months (range: 4-64 months). Out of the 168 patients treated with TACE, 66 (39.2\%) died during the study period

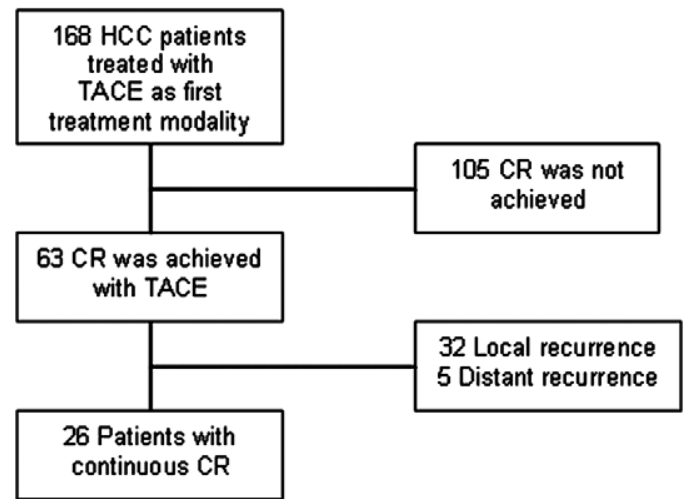

Fig. 1. Flow-chart of the patients included in the study

and 9 patients were lost to follow-up. The overall 1-, 2-, and 3 -year cumulative survival rates of the study population were $88.5 \%, 66.5 \%$ and $48.8 \%$, respectively.

Patients with CR after TACE had higher survival rates than patients without CR (Fig. 2A). In patients who achieved CR versus patients without $\mathrm{CR}$, the cumulative survival rates were $97.6 \%$ versus $83 \%$ after 1 year, $85 \%$ versus $52.8 \%$ after 2 years, and $72.1 \%$ versus $34.3 \%$ after 3 years. Eleven patients (17.4\%)

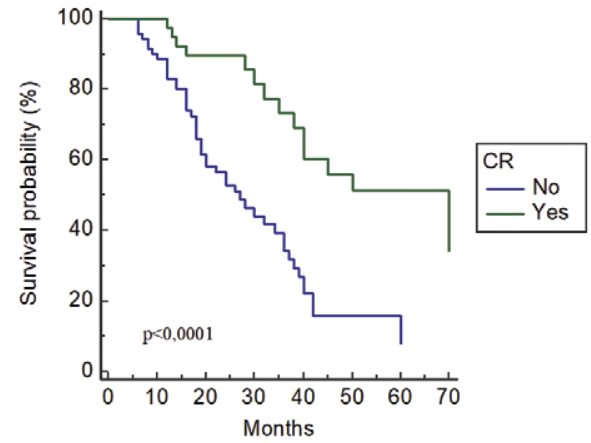

Fig. 2. A. Increased survival rate of HCC patients who achieved CR (green line) compared to that of HCC patients without CR (blue line) $(\mathrm{p}<0.001)$.

Table III. Predictive factors for complete response

\begin{tabular}{lcccccc}
\hline Variable & \multicolumn{3}{c}{ Univariate analysis } & \multicolumn{3}{c}{ Multivariate analysis } \\
\hline & HR & $95 \%$ CI & p-value & HR & $95 \%$ CI & p-value \\
\hline Age $<62$ yr & 1.125 & $0.5488-2.3044$ & 0.749 & & & \\
HBV & 0.988 & $0.3462-2.8190$ & 0.982 & & & \\
HCV & 0.652 & $0.2051-2.0721$ & 0.468 & & & \\
Male sex & 1.060 & $0.5362-2.0967$ & 0.866 & & & \\
MELD score $>10$ & 0.686 & $0.3430-1.3700$ & 0.285 & & & \\
DEBDOX & 1.627 & $0.8077-3.2774$ & 0.173 & & & \\
Absence of PVT & 1.192 & $0.4235-3.3520$ & 0.739 & & & \\
AFP $<22$ ng $/ \mathrm{mL}$ & 3.358 & $1.2300-4.4932$ & 0.019 & & & \\
Tumor size $<$ & 4.424 & $1.8346-10.6703$ & 0.002 & 2.352 & $1.775-3.872$ & 0.022 \\
$4.5 \mathrm{~cm}$ & & & & & & \\
Single nodule & 5.068 & $2.2703-11.3144$ & $<0.001$ & 3.964 & $1.735-9.981$ & $<0.001$ \\
\hline
\end{tabular}

AFP: alpha-fetoprotein; DEBDOX: Doxorubicin-loaded DC Bead; HBV: hepatitis B virus; HCV: hepatitis C virus; MELD: model for end-stage liver disease; PVT: portal vein thrombosis. 


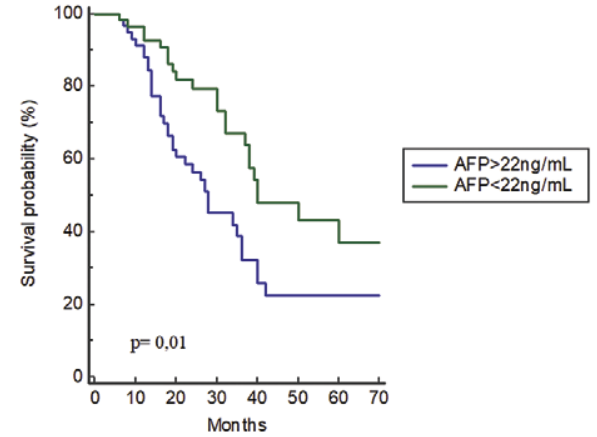

Fig. 2. B. Increased survival rate of HCC patients with normal serum $\alpha$-fetoprotein (AFP) $(\leq 22 \mathrm{ng} /$ $\mathrm{mL}$ ) levels (green line) compared to that of HCC patients with elevated serum AFP $(>22 \mathrm{ng} / \mathrm{mL})$ levels (blue line) $(\mathrm{p}=0.01)$.

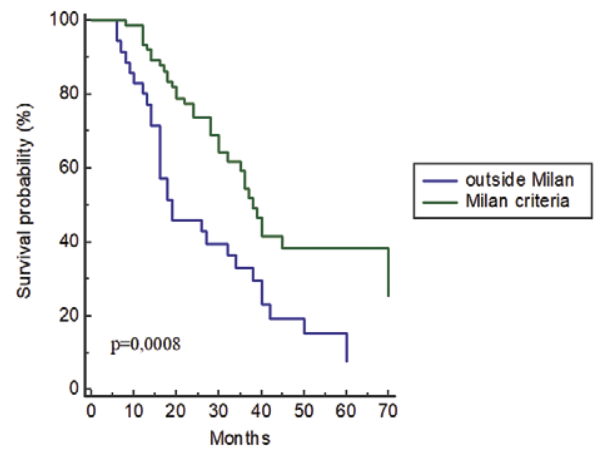

Fig. 2. C. Increased survival rate of HCC patients within the Milan criteria (green line) compared to that of HCC patients outside Milan (blue line) $(\mathrm{p}=0.0008)$.

received a liver transplant after achieving CR. Sorafenib treatment was started in 51 patients $(30.3 \%)$ that had either tumor progression, or hepatic artery damage (2 cases) after repeated sessions of TACE.

Multivariate analysis (Table $\mathrm{V}$ ) revealed that independent predictors for survival were serum AFP $<22 \mathrm{ng} / \mathrm{mL}$ (Fig. 2B), Milan criteria (Fig. 2C), tumor size $<4.5 \mathrm{~cm}$, CR, liver transplantation (Fig. 2D) and absence of PVT (Fig. 2E).

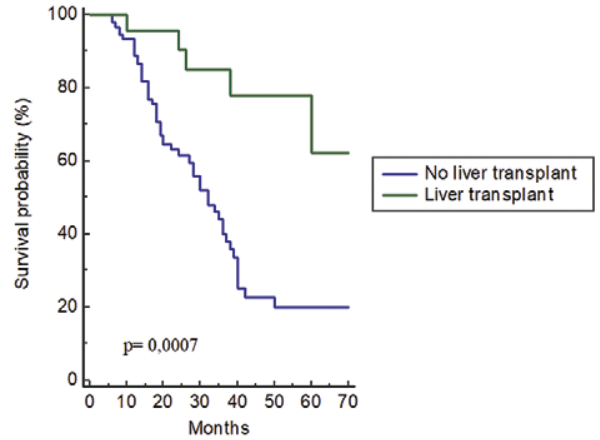

Fig. 2. D. Increased survival rate of HCC patients in whom liver transplant was performed (green line) compared to that of HCC patients without a liver transplant (blue line) $(\mathrm{p}=0.0007)$.

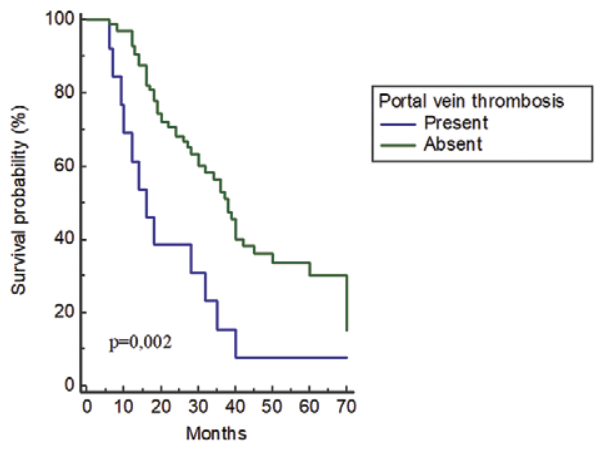

Fig. 2. E. Increased survival rate of HCC patients without PVT (green line) compared to that of HCC patients with PVT (blue line) $(\mathrm{p}=0.002)$.

\section{DISCUSSION}

Hepatocellular carcinoma, one of the most common cancers worldwide, is associated with a high mortality and its incidence rate is still increasing [14]. Curative therapies such as hepatic resection, ablation therapy, or liver transplantation are feasible in only a minority of patients. According to all current guidelines, TACE is the most commonly used treatment in

Table IV. Predictive factors for recurrence

\begin{tabular}{lcccccr}
\hline Variable & \multicolumn{3}{c}{ Univariate analysis } & \multicolumn{3}{c}{ Multivariate analysis } \\
\hline Age $<62$ yr & HR & $95 \%$ CI & p-value & HR & $95 \%$ CI & p-value \\
HCV & 1.511 & $0.5290-4.3136$ & 0.441 & & & \\
HBV & 1.082 & $0.2142-5.4655$ & 0.924 & & & \\
Male sex & 0.802 & $0.1328-4.8438$ & 0.810 & & & \\
MELD score $>10$ & 0.802 & $0.2735-2.3534$ & 0.688 & & & \\
DEBDOX & 1.454 & $0.4580-4.6183$ & 0.525 & & & \\
PVT & 2.029 & $0.6563-6.2724$ & 0.219 & & & \\
AFP $>25$ ng/mL & 0.677 & $0.1254-3.6490$ & 0.649 & & & \\
Tumor size $>4.5$ cm & 3.927 & $1.2964-11.8958$ & 0.013 & 1.650 & $1.453-3.261$ & 0.051 \\
Multiple nodules & 2.745 & $0.5123-8.0989$ & 0.237 & & & \\
\hline AFP: alpha-fetoprotein; DEBDOX: Doxorubicin-loaded DC Bead; HBV: hepatitis B virus; HCV: hepatitis \\
C virus; MELD: model for end-stage liver disease; PVT: portal vein thrombosis &
\end{tabular}


Table V. Predictive factors for survival

\begin{tabular}{lcccccc}
\hline Variable & \multicolumn{3}{c}{ Univariate analysis } & \multicolumn{2}{c}{ Multivariate analysis } \\
\hline Age $<62$ yr & HR & $95 \%$ CI & p-value & HR & $95 \%$ CI & p-value \\
HCV & 1.077 & $0.566-2.046$ & 0.822 & & & \\
HBV & 1.751 & $0.934-3.282$ & 0.081 & & & \\
Male sex & 0.773 & $0.394-1.516$ & 0.454 & & & \\
DEBDOX & 1.546 & $0.777-3.073$ & 0.215 & & & \\
MELD score $<10$ & 1.611 & $0.768-3.378$ & 0.207 & & & \\
Sorafenib & 0.998 & $0.538-1.849$ & 0.994 & & & \\
Single nodule & 2.174 & $1.000-4.744$ & 0.050 & & & \\
AFP $<22$ ng/mL & 1.945 & $1.014-3.683$ & 0.043 & & & \\
Milan criteria & 2.056 & $1.096-3.854$ & 0.025 & 1.572 & $1.242-3.278$ & 0.049 \\
Tumor size $<4.5 \mathrm{~cm}$ & 5.317 & $2.618-9.798$ & 0.001 & 1.978 & $1.798-4.901$ & 0.042 \\
CR & 4.415 & $2.154-9.050$ & 0.001 & 1.742 & $1.415-2.894$ & 0.027 \\
Liver transplant & 3.864 & $1.850-8.068$ & 0.003 & 1.752 & $1.392-3.936$ & 0.005 \\
Absence of PVT & 7.218 & $1.535-9.590$ & 0.005 & 1.291 & $1.056-2.475$ & 0.043 \\
\hline
\end{tabular}

AFP: alpha-fetoprotein; CR: complete response; DEBDOX: Doxorubicin-loaded DC Bead; HBV: hepatitis B virus; HCV: hepatitis C virus; MELD: model for end-stage liver disease; PVT: portal vein thrombosis

patients affected by HCC who have unresectable disease at presentation. In the present study, we evaluated the predictive factors for CR, recurrence after CR and overall survival in naive HCC patients who received TACE as first therapy.

A single HCC nodule represents an indication for curative treatment, as first line therapy [15]. Of our patients, $64.2 \%$ had a single nodule, but despite the early diagnosis, a curative method as a first line therapy was not feasible. The main reasons were tumor location and thrombocytopenia for RFA treatment and the degree of portal hypertension combined with the impossibility of a minimally invasive approach for surgical resection.

In our patients, single nodularity and tumor size $\leq 4.5 \mathrm{~cm}$ were predictive factors for CR. An explanation could be that in case of a single nodule, occlusion of the arteries supplying the tumor by TACE is more effective when compared to the complex tumor vascular feeding routes that are encountered in patients with multinodular HCC.

The correlation between tumor size and CR has been previously demonstrated in several papers [16-18]. Golfieri et al. [17] reported that HCC nodules $\leq 5 \mathrm{~cm}$ were the best responders to TACE with a CR rate of $87.7 \%$ compared with only $25 \%$ in patients with larger nodules $(>5 \mathrm{~cm})$. In the same study there was no significant difference in the CR rate in patients with small nodules $(\leq 2) \mathrm{cm}$ compared to patients with nodules between 2.1 and $5 \mathrm{~cm}$ (68\% vs. 64\%) as it was the case in our study.

Tumor recurrence after TACE-achieved CR represents a significant problem, high rates till $60 \%$ being reported at 1 year after the procedure [19]. Therefore, early diagnosis of HCC recurrence and selection of the appropriate therapy are determining factors that might increase patient survival rates.

Previous reported risk factors associated with HCC recurrence were: location, size and number of HCC nodules, tumor markers, viral infection, age, and liver function [20-22].
We found that multiple nodules and elevated serum AFP levels (> $25 \mathrm{ng} / \mathrm{mL}$ ) were predictive factors for recurrence after CR. Multiple tumors were significantly associated with a low rate of CR and with high risk of recurrence in patients who achieved CR.

In patients with multiple HCC tumors, the higher rate of tumor recurrence cu explained by the fact to the fact that TACE cannot target small undetected satellite lesions, and microscopic remnant tumors may not be detectable by imaging studies during the early phase after TACE procedure [23]. It was shown that even in the presence of complete necrosis on imaging, cancer cells were found around the fibrous capsules surrounding tumors in the tissues that were surgically removed [24]. These findings clearly showed the limitations of the radiological evaluation of tumor response and the fact that early local recurrence could develop and be detectable over time.

According to Rou et al. [25], tumor lesions not detected by CT, but identified using TACE were reported in up to $45 \%$ of cases. Thus, closer surveillance should be performed in patients with multinodular HCC even if they have radiologic remission after TACE.

A high serum AFP level in HCC patients is considered a marker for poor tumor differentiation grade [26]. Riaz et al. [27] reported AFP response after loco-regional therapy as a method of assessing tumor response. We found that a high initial serum AFP level $(>25 \mathrm{ng} / \mathrm{ml})$ was predictive for recurrence.

The Assessment for Retreatment with TACE (ART) score is considered a potentially useful biomarker for safety as well as efficacy, with a strong relationship with survival in HCC patients treated with TACE $[28,29]$. We did not use the ART score as it is not included in the current guidelines, and several newer studies [30-32] demonstrated a lack of external validity and questioned its clinical reliability. 
The OS rates of the entire patient population, excluding patients who underwent LT considered long-term survivors, were at 1- and 3-year $88.7 \%$ and $40.1 \%$, respectively. Similar survival rates were observed in an Italian cohort reporting 1 - and 3-year survival rates of $85 \%$ and $50 \%$, respectively in TACE treated patients for a single HCC nodule [33]. Although the Italian series had a large number of Child Pugh B patients with single nodule HCC, as opposed to our cohort which included almost only Child Pugh A patients, the survival rate was similar in both groups. The explanation might be that in our group $35.7 \%$ of the patients had a multinodular disease, with a negative impact on survival.

As predictive factors for OS, Milan criteria, elevated serum AFP $(>22 \mathrm{ng} / \mathrm{mL})$ level, tumor size $>4.5 \mathrm{~cm}$, multiple nodules and PVT were found in our series to be significant. Tumor size $>4.5 \mathrm{~cm}$, multiple nodules, and elevated serum AFP level were predictive factors for both lack of $\mathrm{CR}$ and recurrence after $\mathrm{CR}$ in our patient group.

Several papers from Asia reported that multinodularity was the most strong predictor for recurrence and survival after TACE $[34,35]$. Our study is the first one from Central and Eastern Europe that evaluates OS and the clinical determinants of recurrence and survival in TACE treated patients.

Non-malignant PVT in patients with HCC represents a therapeutic challenge and was considered a contraindication for TACE due to the risk of liver necrosis or severe function deterioration [36]. Recent studies demonstrated that TACE was a safe treatment option for HCC patients with PVT and has a survival benefit over conservative treatment when performed in a selective manner $[37,38]$. In our cohort we included a limited number of patients with partial nonmalignant PVT $(n=17)$. Despite the fact that none of those patients developed acute liver decompensation after TACE and the presence of PVT was not a predictive factor for recurrence, there was a significant negative impact on OS. A previous larger series found that PVT was independently associated with lower survival among patients with cirrhosis who were being evaluated for liver transplant [39].

Docaens et al. [40] reported that TACE before liver transplant did not prolong long-term survival. The role of TACE as a bridging therapy for HCC patients on the waiting list is controversial [41, 42]. In our cohort, liver transplant after TACE significantly increased survival $(\mathrm{p}=0.0007)$. Patients treated with TACE should be sent for liver transplant evaluation even if they had achieved CR, due to the recurrence risk after TACE on the long term and decreased survival without transplantation.

Previously, super-selective embolization with DEBDOX was considered a potential curative treatment, with better results compared with the cTACE procedure $[43,44]$. We found no significant difference in CR, recurrence or cumulative survival rates between the two groups.

Treatment with Sorafenib was found to be marginally correlated with OS only in the univariate analysis $(\mathrm{p}=0.05)$. This might be due to the relatively small number of cases receiving Sorafenib. These patients with treatment failure after repeated TACE started Sorafenib later, at the time of their dropout from the liver transplant waiting list, due to HCC progression: those patients had a more advanced disease, probably more aggressive tumors and a worse prognosis.

The limitations of this study include its retrospective nature and a single center data collection. Although TACE is recommended to be used in patients with HCC diagnosed at an intermediate BCLC stage, we included patients with early-stage HCC not suitable for resection, RFA or liver transplantation because of old age, inaccessible tumor location for surgery or RFA, or a long waiting time before receiving a donor liver. According to the recently proposed "stage migration strategy" a TACE procedure is often performed outside the current treatment guidelines in a significant percentage of patients with a single nodule [45].

As age of HCC patients gradually increases, more patients would be excluded when considering the treatment guideline algorithm of HCC due to the presence of complications and poor performance status. There is a need to better define patient populations who have the highest chances of achieving $\mathrm{CR}$ and those that have a risk for recurrence.

\section{CONCLUSIONS}

We found that tumor size $\leq 4.5 \mathrm{~cm}$ and single nodularity were predictive factors for $\mathrm{CR}$, and multiple nodules and elevated serum AFP level $(>25 \mathrm{ng} / \mathrm{mL}$ ) were predictive factors for recurrence after $\mathrm{CR}$ in patients who received TACE as a first therapy. Increased initial serum AFP $>22 \mathrm{ng} / \mathrm{mL}$, tumor size $>$ $4.5 \mathrm{~cm}$, outside Milan criteria, not receiving a liver transplant and presence of a portal vein thrombosis have an important negative relation with $O S$.

Conflicts of interest: The authors have no affiliations with or involvement in any organization or entity with any financial interest or non-financial interest regarding this study.

Authors' contributions: R.C., A.C., M.G. and L.G designed the study. M.G.. and R.D, performed the TACE procedures; M.G., R.D. and I.L. evaluated the radiological images. L.P. and G.C. evaluated the biological data. C.E., R.C., A.C. and S.I. analysed the data. R.C., A.C and L.G wrote the paper with input from all authors.

\section{REFERENCES}

1. Ferlay J, Shin HR, Bray F, Forman D, Mathers C, Parkin DM. GLOBOCAN 2008 v2.0, Cancer Incidence and Mortality Worldwide: IARC Cancer Base No. 10. Lyon, France: International Agency for Research on Cancer; 2010.

2. Forner A, Reig ME, de Lope CR, Bruix J. Current strategy for staging and treatment: the BCLC update and future prospects. Semin Liver Dis 2010;30:61-74. doi:10.1055/s-0030-1247133

3. Cauchy F, Fuks D, Belghiti J. HCC: current surgical treatment concepts. Langenbecks Arch Surg 2012;397:681-695. doi:10.1007/s00423-0120911-2

4. Kohles N, Nagel D, Jüngst D, Durner J, Stieber P, Holdenrieder S. Prognostic relevance of oncological serum biomarkers in liver cancer patients undergoing transarterial chemoembolization therapy. Tumour Biol 2012;33:33-40. doi:10.1007/s13277-011-0237-7 
5. Bruix J, Sherman M; American Association for the Study of Liver Diseases. Management of hepatocellular carcinoma: an update. Hepatology 2011;53:1020-1022. doi:10.1002/hep.24199

6. Llovet JM, Bruix J. Systematic review of randomized trials for unresectable hepatocellular carcinoma: chemoembolization improves survival. Hepatology 2003;37:429-442. doi:10.1053/ jhep.2003.50047

7. Lencioni R, de Baere T, Soulen MC, Rilling WS, Geschwind JF. Lipiodol transarterial chemoembolization for hepatocellular carcinoma: a systematic review of efficacy and safety data. Hepatology 2016;64:106116. doi:10.1002/hep. 28453

8. European Association for the Study of the Liver. EASL clinical practice guidelines: management of hepatocellular carcinoma. J Hepatol 2018;69:182-236. doi:10.1016/j.jhep.2018.03.019

9. Hsu KF, Chu CH, Chan DC, et al. Superselective transarterial chemoembolization vs hepatic resection for resectable early-stage hepatocellular carcinoma in patients with Child-Pugh class a liver function. Eur J Radiol 2012;81:466-471. doi:10.1016/j.ejrad.2010.12.058

10. European Association for the Study of the Liver, European Organisation for Research Treatment of Cancer. EASL-EORTC clinical practice guidelines: management of hepatocellular carcinoma. J Hepatol 2012;56:908-943. doi:10.1016/j.jhep.2011.12.001

11. Llovet JM, Brú C, Bruix J. Prognosis of hepatocellular carcinoma: The BCLC staging classification. Semin Liver Dis 1999;19:329-338. doi:10.1055/s-2007-1007122

12. Piscaglia F, Gianstefani A, Ravaioli M, et al; Bologna Liver Transplant Group. Criteria for diagnosing benign portal vein thrombosis in the assessment of patients with cirrhosis and hepatocellular carcinoma for liver transplantation. Liver Transpl 2010;16:658-667. doi:10.1002/ 1t.22044

13. Lencioni R, Llovet JM. Modified RECIST (mRECIST) assessment for hepatocellular carcinoma. Semin Liver Dis 2010;30:52-60. doi:10.1055/s-0030-1247132

14. Venook AP, Papandreou C, Furuse J, de Guevara LL. The incidence and epidemiology of hepatocellular carcinoma: a global and regional perspective. Oncologist 2010;15:5-13. . doi:10.1634/ theoncologist.2010-S4-05

15. Bruix J, Sherman M; Practice Guidelines Committee, American Association for the Study of Liver Diseases. Management of hepatocellular carcinoma. Hepatology 2005;42:1208-1236. doi:10.1002/ hep. 20933

16. Ebied OM, Federle MP, Carr BI, et al. Evaluation of responses to chemoembolization in patients with unresectable hepatocellular carcinoma. Cancer 2003;97:1042-1050. doi:10.1002/cncr.1111

17. Golfieri R, Renzulli M, Mosconi C, et al. Hepatocellular carcinoma responding to superselective transarterial chemoembolization: an issue of nodule dimension? J Vasc Interv Radiol 2013;24:509-517. doi:10.1016/j.jvir.2012.12.013

18. Gattoni F, Cornalba G, Brambilla G, et al. Survival of 184 patients with hepatocellular carcinoma in cirrhotic liver treated with chemoembolization. A multicenter study. Radiol Med 1998;95:362-368.

19. Kinugasa H, Nouso K, Takeuchi Y, et al. Risk factors for recurrence after transarterial chemoembolization for early-stage hepatocellular carcinoma. J Gastroenterol 2012;47:421-426. doi:10.1007/s00535-011-0492-9

20. Nouso K, Ito Y, Kuwaki K, et al. Prognostic factors and treatment effects for hepatocellular carcinoma in Child C cirrhosis. Br J Cancer 2008;98:1161-1165. doi:10.1038/sj.bjc.6604282

21. Ueno S, Tanabe G, Nuruki K, et al. Prognosis of hepatocellular carcinoma associated with Child class B and C cirrhosis in relation to treatment: a multivariate analysis of 411 patients at a single center. J Hepatobiliary Pancreat Surg 2002;9:469-477. doi:10.1007/s005340200058

22. Arimura E, Kotoh K, Nakamuta M, Morizono S, Enjoji M, Nawata H. Local recurrence is an important prognostic factor of hepatocellular carcinoma. World J Gastroenterol 2005;11:5601-5606. doi:10.3748/ wjg.v11.i36.5601

23. Park W, Chung YH, Kim JA, et al. Recurrences of hepatocellular carcinoma following complete remission by transarterial chemoembolization or radiofrequency therapy: focused on the recurrence patterns. Hepatol Res 2013;43:1304-1312. doi:10.1111/hepr.12083

24. Raby N, Karani J, Michell M, Gimson A, Nunnerley H, Williams R. Lipiodol enhanced CT scanning in assessment of hepatocellular carcinoma. Clin Radiol 1989;40:480-485. doi:10.1016/S00099260(89)80254-5

25. Rou WS, Lee BS, Moon HS, Lee ES, Kim SH, Lee HY. Risk factors and therapeutic results of early local recurrence after transcatheter arterial chemoembolization. World J Gastroenterol 2014;20:6995-7004. doi:10.3748/wjg.v20.i22.6995

26. Okuda H, Nakanishi T, Takatsu K, et al. Comparison of clinicopathological features of patients with hepatocellular carcinoma seropositive for alpha-fetoprotein alone and those seropositive for des-gamma-carboxy prothrombin alone. J Gastroenterol Hepatol 2001;16:1290-1296. doi:10.1046/j.1440-1746.2001.02610.x

27. Riaz A, Ryu RK, Kulik LM, et al. Alpha-fetoprotein response after locoregional therapy for hepatocellular carcinoma: oncologic marker of radiologic response, progression, and survival. J Clin Oncol 2009;27:5734-5742. doi:10.1200/JCO.2009.23.1282

28. Sieghart W, Hucke F, Pinter M, et al. The ART of decision making: retreatment with transarterial chemoembolization in patients with hepatocellular carcinoma. Hepatology 2013;57:2261-2273. doi:10.1002/ hep. 26256

29. Hucke F, Sieghart W, Pinter M, et al. The ART-strategy: sequential assessment of the ART score predicts outcome of patients with hepatocellular carcinoma re-treated with TACE. J Hepatol 2014;60:118126. doi:10.1016/j.jhep.2013.08.022

30. Kudo M, Arizumi T, Ueshima K. Assessment for retreatment (ART) score for repeated transarterial chemoembolization in patients with hepatocellular carcinoma. Hepatology 2014;59:2424-2425. doi:10.1002/ hep. 26760

31. Terzi E, Terenzi L, Venerandi L, et al. The ART score is not effective to select patients for transarterial chemoembolization retreatment in an Italian series. Dig Dis 2014;32:711-716. doi:10.1159/000368007

32. Piscaglia F, Ogasawara S. Patient Selection for Transarterial Chemoembolization in Hepatocellular Carcinoma: Importance of Benefit/ Risk Assessment. Liver Cancer 2018;7:104-119. doi:10.1159/000485471

33. Terzi E, Piscaglia F, Forlani L, et al. TACE performed in patients with a single nodule of Hepatocellular Carcinoma. BMC Cancer 2014;14:601. doi:10.1186/1471-2407-14-601

34. Douhara A, Namisaki T, Moriya K, et al. Predisposing factors for hepatocellular carcinoma recurrence following initial remission after transcatheter arterial chemoembolization. Oncol Lett 2017;14:30283034. doi:10.3892/ol.2017.6489

35. Jeong SO, Kim EB, Jeong SW, et al. Predictive Factors for Complete Response and Recurrence after Transarterial Chemoembolization in Hepatocellular Carcinoma. Gut Liver 2017;11:409-416. doi:10.5009/ gnl16001

36. Kulik LM, Carr BI, Mulcahy MF, et al. Safety and efficacy of $90 \mathrm{Y}$ radiotherapy for hepatocellular carcinoma with and without portal vein thrombosis. Hepatology 2008;47:71-81. doi:10.1002/hep.21980 
37. Kim JH, Yoon HK, Kim SY, et al. Transcatheter arterial chemoembolization vs. chemoinfusion for unresectable hepatocellular carcinoma in patients with major portal vein thrombosis. Aliment Pharmacol Ther 2009;29:1291-1298. doi:10.1111/j.13652036.2009.04016.x

38. Luo J, Guo RP, Lai EC, et al. Transarterial chemoembolization for unresectable hepatocellular carcinoma with portal vein tumor thrombosis: a prospective comparative study. Ann Surg Oncol 2011;18:413-420. doi:10.1245/s10434-010-1321-8

39. Englesbe MJ, Kubus J, Muhammad W, et al. Portal vein thrombosis and survival in patients with cirrhosis. Liver Transpl 2010;16:83-90. doi:10.1002/1t.21941

40. Decaens T, Roudot-Thoraval F, Bresson-Hadni S, et al. Impact of pretransplantation transarterial chemoembolization on survival and recurrence after liver transplantation for hepatocellular carcinoma. Liver Transpl 2005;11:767-775. doi:10.1002/lt.20418

41. Lesurtel M, Müllhaupt B, Pestalozzi BC, Pfammatter T, Clavien PA. Transarterial chemoembolization as a bridge to liver transplantation for hepatocellular carcinoma: an evidence-based analysis. Am J Transplant 2006;6:2644-2650. doi:10.1111/j.16006143.2006.01509.x

42. Terzi E, Ray Kim W, Sanchez W, et al. Impact of multiple transarterial chemoembolization treatments on hepatocellular carcinoma for patients awaiting liver transplantation. Liver Transpl 2015;21:248-257. doi:10.1002/lt.24041

43. Song MJ, Park CH, Kim JD, et al. Drug-eluting bead loaded with doxorubicin versus conventional Lipiodol-based transarterial chemoembolization in the treatment of hepatocellular carcinoma: a case-control study of Asian patients. Eur J Gastroenterol Hepatol 2011;23:521-527. doi:10.1097/MEG.0b013e328346d505

44. Song MJ, Chun HJ, Song DS, et al. Comparative study between doxorubicin-eluting beads and conventional transarterial chemoembolization for treatment of hepatocellular carcinoma. J Hepatol 2012;57:1244-1250. doi:10.1016/j.jhep.2012.07.017

45. Bolondi L, Burroughs A, Dufour JF, et al. Heterogeneity of patients with intermediate (BCLC B) hepatocellular carcinoma: proposal for a subclassification to facilitate treatment decisions. Semin Liver Dis 2012;32:348-359. doi:10.1055/s-0032-1329906 